\title{
Sequence-optimised E2 constructs from BVDV-1b and BVDV-2 for DNA immunisation in cattle
}

\author{
Bernard COUVREUR $^{\mathrm{a}, \mathrm{b} *}$, Carine LETELLIER $^{\mathrm{a}}$, Fabrice OLIVIER ${ }^{\mathrm{a}}$, \\ Pierre DEHAN $^{\mathrm{c}}$, Abdelatif ElOUAHABI ${ }^{\mathrm{d}}$, Michel VANDENBRANDEN ${ }^{\mathrm{d}}$, \\ Jean-Marie RUYSSCHAERT ${ }^{\mathrm{d}}$, Claude HAMERS ${ }^{\mathrm{c}}$, Paul-Pierre PASTORET ${ }^{\mathrm{c}}$, \\ Pierre KERKHOFS ${ }^{\mathrm{a}}$
${ }^{\text {a }}$ Department of Virology, Veterinary and Agrochemical Research Centre, Bruxelles, Belgium
${ }^{\mathrm{b}}$ Present address: Service de Génétique Appliquée, ULB CP 300,
12 rue des professeurs Jeener et Brachet, 6041 Gosselies, Belgique
${ }^{\mathrm{c}}$ Vaccinologie-Immunologie, Université de Liège, Belgium \\ ${ }^{\mathrm{d}}$ Laboratory of Structure and Function of Biological Membranes, ULB, Bruxelles, Belgium
}

(Received 7 September 2006; accepted 14 May 2007)

\begin{abstract}
We report DNA immunisation experiments in cattle using plasmid constructs that encoded glycoprotein E2 from bovine viral diarrhoea virus (BVDV)-1 (E2.1) and BVDV-2 (E2.2). The coding sequences were optimised for efficient expression in mammalian cells. A modified leader peptide sequence from protein $\mathrm{gD}$ of BoHV1 was inserted upstream of the E2 coding sequences for efficient membrane export of the proteins. Recombinant E2 were efficiently expressed in COS7 cells and they presented the native viral epitopes as judged by differential recognition by antisera from cattle infected with BVDV-1 or BVDV-2. Inoculation of pooled plasmid DNA in young cattle elicited antibodies capable of neutralising viral strains representing the major circulating BVDV genotypes.
\end{abstract}

BVDV / DNA immunisation / E2 / neutralisation / synthetic gene

\section{INTRODUCTION}

Bovine viral diarrhoea virus (BVDV) is a positive strand enveloped RNA virus. The genome comprises a single large open reading frame flanked by untranslated regions at the 5' and 3' ends. It is translated into a large unique polyprotein of $\sim 4000$ amino-acids. In infected cells, it is cleaved into structural and non-structural proteins by cellular as well as virus-encoded proteases. Structural proteins are the nucleocapsid protein $\mathrm{C}$, surface glycoproteins E0 or Erns, El and E2 [56].

\footnotetext{
* Corresponding author: bcouvreu@ulb.ac.be
}

BVDV infection is widespread in cattle worldwide and it causes important economic losses [28]. In adult animals, infection is usually mild or subclinical although outbreaks of haemorrhagic disease associated to BVDV have been observed [50]. Transplacental transmission during the first three months of pregnancy causes abortion or the birth of immunotolerant persistently infected (PI) calves. PI animals may look healthy but do not mount a specific immune response and shed enormous amounts of virus. They generally succumb from mucosal disease caused by superinfection by antigenically similar cytopathic 
strains. PI animals are also the main viral reservoir and source of contamination within and between herds [1]. BVDV isolates are classified within BVDV-1 and BVDV-2 after serological cross-reactivity and sequence relatedness. They have the taxonomic status of viral species within the pestivirus genus along with BDV and CSFV [56]. Strains from both species are circulating worldwide but with different distributions [53,57]. They induce similar diseases except that hypervirulent strains are only found within BVDV-2 [50].

Numerous inactivated or attenuated vaccines are available worldwide. Most induce horizontal protection and some claim to induce vertical protection as well [32]. In this context, DNA vaccination seems to be a promising approach. Indeed, in the early nineteen-nineties researchers first observed that plasmid constructs expressing an antigenic protein elicited an immune response after mere intramuscular or intradermal injection. Plasmid constructs are easier to develop, produce and store than conventional or subunit vaccines. Furthermore, DNA often favours the elicitation of a type 1 cellular response that is immunologically favourable to the resolution of viral infections [43]. Finally, the versatility of the technology makes it possible to design multivalent vaccines from variants of a given pathogen or from different pathogens as well as to devise strategies to differentiate infected from vaccinated animals. On the contrary, subunit vaccines are more complex to develop since they depend on efficient expression systems, protein purification and stabilised storage. They do, however, alleviate public concerns about genetically modified organisms. In any case, the prerequisites for successful vaccine design, are the choice of the appropriate target antigen or antigens and high expression levels of the recombinant protein or proteins.

In cattle, resolution of BVDV infection is associated with neutralising anti- bodies [30] and with a cellular response due to CD4+ T-lymphocytes [29]. CD4+ T cells from recovering calves are induced to proliferate by structural proteins Erns, E2, $\mathrm{C}$ and by non structural proteins NS2-3 and Npro [13, 14].

The major surface glycoprotein E2 is the main target of neutralising antibodies [6]. Neutralising antibodies and a cellular response are produced in animals vaccinated with various recombinant viruses expressing E2 [3, 17,21], with DNA plasmid constructs expressing E2 [26, 42, 45] or with E2 subunit vaccines [7, 11, 12,39, 58]. Purified recombinant E2 from BVDV1a strain Singer lead to partial protection against acute infection by the homologous strain [7]. Another E2 subunit vaccine was shown to induce partial fetal protection in a sheep model of BVDV transplacental transmission $[11,12]$.

This makes E2 a prime candidate antigen for subunit or DNA vaccination. However, the protein sequence is variable and cross-neutralising activity as well as crossreactive cellular response are often poor or absent between viral strains from BVDV-1 and BVDV-2 [11,26]. Also, a subunit E2 vaccine from one genotype does not protect from challenge with a strain from the other genotype [7,11].

In order to develop a DNA vaccine against the wider spectrum of circulating BVDV strains, we constructed recombinant plasmids expressing the E2 proteins from field strains of the two main BVDV genotypes i.e. BVDV-1b and BVDV-2. Besides, BVDV- $1 \mathrm{~b}$ is the most frequent subtype in Belgium $[15,41]$, in continental Europe and probably also worldwide [53, 57]. The coding sequences were optimised for expression in mammalian cells and inserted under the control of the strong CMV promoter in plasmid pCDNA3. Both E2 proteins were efficiently expressed in vitro and presented native epitopes. BVDV-free calves were then immunised with plasmid DNA via the intramuscular 
(IM), intradermal (ID) and intranasal (IN) routes. They developed neutralising antibodies against viral strain representatives of BVDV-1 and BVDV-2.

\section{MATERIALS AND METHODS}

\subsection{Virus and cells}

The BVDV-1b field strain CP1945 and the BVDV-2 field strain WVD829 had been previously isolated from leucocytes of PI animals at VAR and typified [15,41]. They did not cause any discernible symptoms in BVDV-free bulls (unpublished observations). NADL and Osloss strains as well as strain 890 were used in neutralisation experiments as representatives for BVDV genotypes Ia, Ib and II, respectively. Recent field isolates Culi4 (BVDV1d), L256 (BVDV-1e), WVD829 (BVDV2) and BSE 921 (BVDV-2) were included too $[15,41]$. All strains but NADL are noncytopathogenic to cell cultures.

Virus titration, isolation and seroneutralisation studies were performed on the Madin Darby Bovine Kidney (MDBK) cell line, cultured with minimum essential medium, supplemented with $10 \%$ irradiated foetal calf serum, $1 \%$ non-essential Amino Acids, 100 ui $/ \mathrm{mL}$ PenicillinStreptomycin, $50 \mu \mathrm{g} / \mathrm{mL}$ gentamicin and incubated at $37{ }^{\circ} \mathrm{C}$ under $5 \% \mathrm{CO}_{2}$. Culture media and supplements were all from Gibco BRL Life Technologies (Gaithersburg, MD, USA).

\subsection{Molecular biology techniques}

Total RNA was extracted from BVDVinfected MDBK cells using Trizol (Life Technologies). The RNA was reversetranscribed with the Superscript II system (Life Technologies) using random hexamers as primers (Roche Molecular Biochemicals, Indianapolis, IN, USA). All PCR experiments were performed using the Expand High-Fidelity PCR system (Roche
Molecular Biochemicals) in $100 \mu \mathrm{L}$ final volume with $1.5 \mathrm{mM} \mathrm{MgCl}{ }_{2}$ and thermal cycler TC1 (Perkin Elmer Applied Biosystems, Lincoln, CA, USA). PCR products were routinely inserted into pCR3.1 by the T/A cloning method (Invitrogen, Carlsbad, CA, USA). Primers are listed in Table I.

Constructs were subcloned into plasmid pCDNA3 (Invitrogen) using restriction enzymes and the rapid DNA ligation kit from Roche Molecular Biochemicals.

Inserts were fully sequenced on both strands by the dye terminator method and the reaction products were run onto an $\mathrm{ABI}$ PRISM model 310 genetic analyser (PE Applied Biosystems).

All commercial products were used according to the manufacturers' instructions.

\subsection{Computer-assisted analysis of sequences}

Algorithms for the management of sequencing projects, translation, backtranslation, determination of restriction sites and calculation of codon usage were from the Wisconsin Package Version 10.1 (Genetics Computer Group, GCG, Madison, Wisconsin, USA).

The presence of the instability motif ATTTA was determined by visual examination of the nucleotide sequences. This motif has been shown to confer rapid degradation to mammalian messenger RNA [5]. Putative polyadenylation signals were searched online with the POLYAH $^{1}$ programme. RNA splice sites were searched using the Netgene 2 programme [10]. Probable cellular targeting and the eukaryotic leader peptide cleavage site were determined with the Target $\mathrm{P}$ and Signal $\mathrm{P}$ programmes, respectively [44]. Transmembrane helixes were searched with the TMHMM version 2.0 programme [37]. These four programmes

\footnotetext{
${ }^{1}$ http://www.softberry.com/berry.phtml
} 
Table I. Oligonucleotide primers for the cloning of E2 coding sequences. Start and stop codons are printed in bold. The stop codon TAA is generated in GP53R1B when the Taq polymerase adds a non-template A at the 3' end of amplicon E2.1. The parts of primers that actually anneal to the viral cDNA are underlined. Restriction sites in primers are italicized.

\begin{tabular}{|c|c|c|c|c|}
\hline Primer & Sequence (5' to 3') & $\begin{array}{c}\text { Anneals } \\
\text { to position }\end{array}$ & $\begin{array}{l}\text { PCR product } \\
\text { (size in bp) }\end{array}$ & Substrate \\
\hline GP53F1 (fwd) & AAGCTTTAAGGATGATACTCATAACAGGAGC & $2431-2452^{\mathrm{a}}$ & E2.1 (1162) & cDNA from CP1945- \\
\hline GP53R1B (rev) & ATCCCGAGGTCATTTGTTCTGATA & $3562-3584^{\mathrm{a}}$ & & infected MDBK cells \\
\hline GP53F2 (fwd) & AAGCTTCCATAATGCTAATAACAGGGGCAC & $2438-2456^{\mathrm{b}}$ & E2.2 (1139) & cDNA from WVD829- \\
\hline GP53R2 (rev) & СТAACCCATAGCCATCTGCTCAG & $3558-3577^{\mathrm{b}}$ & & infected MDBK cells \\
\hline LP2 (rev) & CTGGGCCATGGTGGCCACGGCGAGCAGCGCGCCCA & NA & & \\
\hline
\end{tabular}

${ }^{\text {a }}$ On the genome sequence of strain Osloss [16].

${ }^{\mathrm{b}}$ On the genome sequence of strain 890 [49]. NA, not applicable. 
were used online at the Centre for Biological Analysis of the Technical University of Denmark ${ }^{2}$.

\subsection{Cloning and expression of BVDV- 1 and BVDV-2 major surface glycoprotein E2}

The E2 coding sequences from BVDV1b strain CP1945 and BVDV-2 strain WVD829 were amplified by PCR. Primers (Tab. I) were chosen so as to amplify a portion of the viral genome that encodes the last 7-9 residues of the $\mathrm{C}$-terminal hydrophobic region of glycoprotein $\mathrm{E} 1$, the E1-E2 cleavage site and the complete glycoprotein E2 up to its cleavage site from protein P7. Limits between the putative structural proteins within the polyprotein were determined after Becher et al. [4] and Rümenapf et al. [51]. 5' primers were designed from previous partial sequences of the E1-E2 coding region [15]. 3' primers were designed from multiple alignments of published BVDV sequences. For both primer pairs, cycling conditions were the following: $94^{\circ} \mathrm{C}$ for $2 \mathrm{~min}$ then 25 cycles of $94{ }^{\circ} \mathrm{C}$ for $30 \mathrm{~s}, 51{ }^{\circ} \mathrm{C}$ for $60 \mathrm{~s}, 72{ }^{\circ} \mathrm{C}$ for 2 min with $20 \mathrm{~s}$ extension starting at cycle 10 , and a final incubation of $72{ }^{\circ} \mathrm{C}$ for $7 \mathrm{~min}$.

Next, expression-optimised coding sequences were designed. RNA splice sites, internal polyadenylation motifs and instability motifs were removed. Since codon usage bias is identical in the mouse, human and bovine (not shown), the codon frequency was also changed after highly expressed human genes [22] while keeping the native peptide sequence. Briefly, least used codons were changed to most used codons so that the overall codon usage profile became similar to the one of highly expressed human genes. The coding sequence ended with two stop codons in order to avoid production of "run-off" proteins. The actual synthetic genes were

\footnotetext{
${ }^{2}$ http://www.cbs.dtu.dk/services/
}

constructed by GENEART GmbH (Regensburg, Germany).

The protein encoded by the synthetic genes started four residues upstream from the E1-E2 cleavage site so that signal cleavage would reproduce the predicted original N-terminus of E2: YPDCK for CP1945 and FPECK for WVD829. The E2 synthetic genes were subcloned into pCDNA3 after Hind 3 and XhoI enzymatic restriction, thus creating constructs $\mathrm{pCMV}$ synE2.1 and pCMV-synE2.2 respectively.

The coding sequence for the leader peptide of surface protein $\mathrm{gD}$ from BoHV-1 was amplified with primers LP1 and LP2 (Tab. I). The forward primer LP1 was modified to include an ATG codon in Kozak's consensus for efficient initiation of translation [33]. Cycling conditions were $94{ }^{\circ} \mathrm{C}$ for $2 \mathrm{~min}$, then 25 cycles of $94{ }^{\circ} \mathrm{C}$ for $30 \mathrm{~s}, 60{ }^{\circ} \mathrm{C}$ for $30 \mathrm{~s}$ and $72{ }^{\circ} \mathrm{C}$ for $30 \mathrm{~s}$. The PCR product was digested with Hind 3 and Sfil and ligated into pCMV-synE2.1 and pCMV-synE2.2 at the corresponding restriction sites in the frame at the 5' end of the E2 coding sequences thus creating pLP-synE2.1 and pLP-synE2.2 respectively.

\subsection{Transfection experiments}

Plasmid DNA was routinely purified from $\mathrm{DH} 5 \alpha$ or TOP10F' bacteria cells by the quantum prep method (Biorad, Hercules, CA, USA). Sub-confluent COS cells in 24-well plates were transfected in duplicate with 300 to 600 ng DNA per well using lipofectamine (Invitrogen). The cells were washed and fixed at $24 \mathrm{~h}, 48 \mathrm{~h}$ and $72 \mathrm{~h}$ post transfection to be tested for recognition of viral proteins by bovine anti-BVD sera using the immunoperoxydase monolayer assay (IPMA) [36].

Anti-BVDV sera had been previously collected from BVDV-free calves experimentally infected with selected genotyped BVDV strains $[23,24,41]$. They were routinely diluted $1 / 300$ in PBS- $0.1 \%$ NaN3. 
We also investigated the cellular localisation of the two E2 glycoproteins. In this research group, the results of investigations on the localisation of cloned BoHV-1 structural proteins using a modified IPMA were consistent with confocal microscopy [54]. Therefore, we considered the former as a reliable approach. COS cells in 24-well plates were transiently transfected with plasmid constructs expressing gD (pCI-gD) [54], protein $\mathrm{N}$ (pNsyn) [8] and our new constructs pCMVLPsynE2.1 and pCMVL-PsynE2.2. After $48 \mathrm{~h}$, half the culture plates were first dried then frozen at $-20{ }^{\circ} \mathrm{C}$ and finally fixed with $4 \%$ formaldehyde, leading to permeabilisation of membranes (permeabilised condition). In the other half, we avoided permeabilisation by staining life cells and by avoiding detergent in all buffers (non-permeabilised condition). Cell cultures were then incubated with the respective specific anti-gD, anti-N and anti-E2 bovine antisera. Non-transfected controls were incubated with the anti-E2 antiserum only.

\subsection{Large-scale production of plasmid DNA for DNA immunisation}

Large amounts of plasmid DNA were produced by alkaline lysis of overnight DH5 $\alpha$ bacterial cultures followed by affinity chromatography on anion-exchange resin (Plasmid Giga kit, Qiagen, Hilden, Germany). Columns were regenerated and re-used up to four times as described by Gregory et al. [20]. Typically, lysates from two $2.5 \mathrm{~L}$ cultures were each passaged twice through a single column. The DNA was eluted in sterile bi-distilled water. Each individual batch or passage was quantitated by spectrometry and gel electrophoresis and tested for expression in COS cells. First passage batches typically produced 8 to $12 \mathrm{mg}$ DNA whereas second passages produced $\sim 50 \%$ of first passage yields. No consistent differences in purity or in expression levels were observed between batches. Therefore, batches for a given construct were pooled before inoculation.

\subsection{DNA immunisation in cattle}

All manipulations were conducted in isolation facilities. Danish crossbred male calves, negatives for BVDV virus and antibodies were purchased from Husdyrformidling Syd (Rødekro, Denmark). The animals were maintained and handled according to local and national ethical guidelines.

DNA for the IN route was made into a cationic liposome DNA complex by timely mixing up the DNA with protamine (Sigma) and diC(14)-amidine (vectamidine $^{\mathrm{TM}}$, Biotech Tools, Brussels, Belgium) following a 1:0.6:4 molecular ratio after Elouahabi et al. [18]. Intranasal instillation was performed by dropping the DNA-protamine-vectamidine complexes in $7.5 \mathrm{~mL}$ saline into both nostrils. For the IM inoculation route, 2.5 to $2.75 \mathrm{~mL}$ of DNA solution was injected into a single site located at the base of the neck. The ID route consisted in injecting 1.1 to $1.3 \mathrm{~mL}$ into 4 to 7 points into the shaven and disinfected (isobetadine) skin of the neck.

Three bulls aged $\sim 8.5$ months each received 3 injections of $1 \mathrm{mg}$ plasmid DNA consisting in $500 \mu \mathrm{g}$ pLP-synE2.1 and $500 \mu \mathrm{g}$ pLP-synE2.2 in sterile endotoxinfree PBS (Sigma, St. Louis, MO, USA). In the first injection, the DNA dose was fractionated into three inoculums: (1) intramuscular: $333 \mu \mathrm{g}$, (2) intradermal: $333 \mu \mathrm{g}$, (3) intranasal: $333 \mu \mathrm{g}$. At day 21 , the animals were given a first booster of the same material by the IM $(500 \mu \mathrm{g})$ and ID $(500 \mu \mathrm{g})$ routes only. A second, identical booster was given at day 69 . A fourth bull was kept not inoculated to monitor environmental contamination by pestiviruses. Blood samples were taken at the days of inoculation, two weeks after the 
Table II. Features of the E2 coding sequences.

\begin{tabular}{|c|c|c|c|c|c|c|c|}
\hline \multirow[t]{2}{*}{ Coding sequence } & \multirow{2}{*}{$\begin{array}{c}\text { Accession } \\
\text { numbe }\end{array}$} & \multirow{2}{*}{$\begin{array}{l}\text { Size } \\
\text { (bp) }\end{array}$} & \multicolumn{2}{|c|}{ Splice sites } & \multirow{2}{*}{$\begin{array}{l}\text { Other } \\
\text { motifs }\end{array}$} & \multirow{2}{*}{$\begin{array}{c}\% \\
\text { GC }\end{array}$} & \multirow{2}{*}{$\begin{array}{l}\text { Expression } \\
\text { in COS cells }\end{array}$} \\
\hline & & & Donor & Acceptor & & & \\
\hline Native type 1 E2 & AJ715391 & 1152 & 5 & 7 & ins. $(2 \mathrm{X})$ & 46 & No \\
\hline Native type 2 E2 & AJ715392 & 1143 & 7 & 5 & polyAd., ins. & 44 & No \\
\hline Synthetic type 1 E2 & AJ715393 & 1134 & 0 & 0 & None & 59 & Strong \\
\hline Synthetic type 2 E2 & AJ715394 & 1128 & 0 & 0 & None & 57 & Strong \\
\hline
\end{tabular}

polyAd., Polyadenylation signal; ins., instability motif ATTTA.

first and second injections then every week for 1.5 months.

\subsection{Assessment of the humoral response}

Sera from all calves were tested for neutralising antibodies against viral strain representatives of the three main BVDV genotypes: Osloss (nc) for BVDV-1b; NADL (cp) for BVDV-1a and 890 (nc) for BVDV2. Additional experiments were conducted on strains Culi4 (1d), L256 (1e), WVD829 (2) and BSE921 (2) recently isolated from field samples in Belgium [15,41]. Tests were conducted in microtitre plates containing confluent monolayers of MDBK cells. All sera were tested in duplicate in two-fold dilution, starting at dilution 1:2, against 64 to $128 \mathrm{TCID}_{50}$ of virus. Unneutralised virus was detected by indirect immunofluorescence staining using a pool of monoclonal antibodies as primary antibodies [9].

\section{RESULTS}

\subsection{Cloning and expression of BVDV E2 proteins}

The coding sequences for E2 from field isolates CP1945 (BVDV-1b) and WVD829 (BVDV-2) were isolated by RT/PCR and inserted into the pCR3.1 mammalian expression vector. The new sequences were submitted to the EMBL database and assigned accession numbers AJ715391 and AJ715392 respectively. No viral proteins were detected by IPMA using bovine antisera against BVDV after transfection of COS cells (not shown).

Analysis of the sequences revealed the presence of (i) donor and acceptor RNA splice sites, (ii) an internal polyadenylation motif, (iii) the mRNA instability motif ATTTA (Tab. II). The codon usage was also found to be very different from the average codon usage in mammalian cells (i.e. bovine, murine or human). Therefore, the nucleotide sequences were optimised for expression and synthetic genes were constructed accordingly. They were assigned accession numbers AJ715393 and AJ715394 respectively. As compared to their cognate native sequences, the optimised ones were $84 \%$ identical at the nucleotide level and $100 \%$ identical at the amino-acid level. The $\mathrm{G}$ or $\mathrm{C}$ contents increased from $\sim 45 \%$ to $\sim 58 \%$ (Tab. II). The synthetic genes were then inserted into plasmid pCDNA3.1 to create pCMVsynE2.1 and pCMV-synE2.2. Only the latter gave a weak signal on COS transfected cells after IPMA using a hyperimmune bovine antiserum (Tab. III and Fig. 1).

Secondly, a modified leader peptide sequence from protein $\mathrm{gD}$ of BHV-1 was amplified by PCR. Sequence analysis with Target $\mathrm{P}$ indicated that the leader peptide belonged to the highest reliability class $(\mathrm{RC}=1)$ for a secretory pathway. The nucleotide sequence has $67 \% \mathrm{G}$ or $\mathrm{C}$ and an average mammalian codon usage; it lacked splice sites or instability 


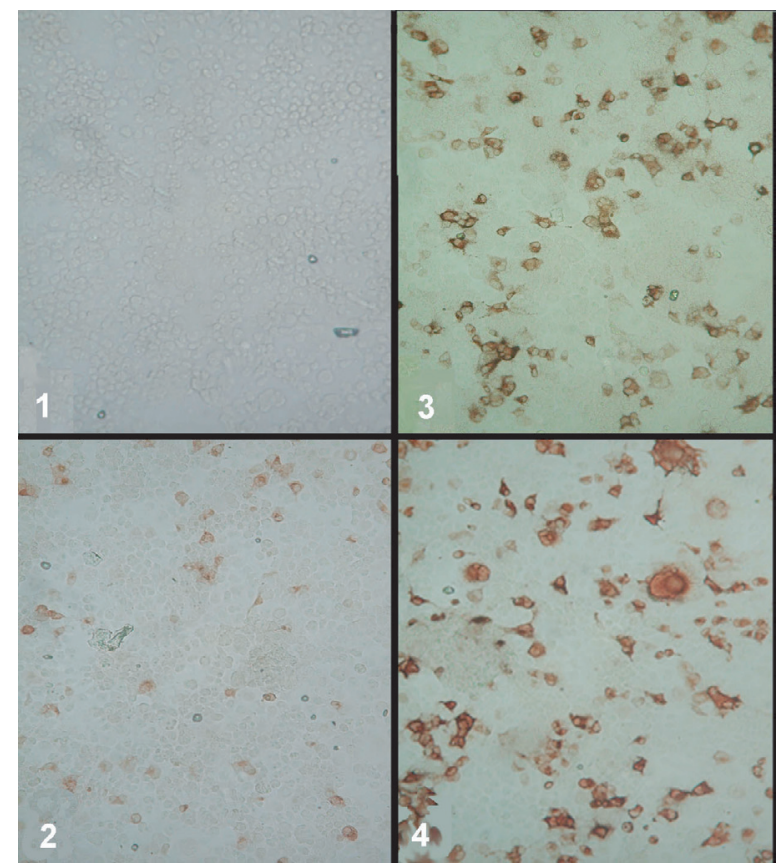

Figure 1. Transient expression of BVDV E2 glycoproteins in Cos7 cells. Cos7 cells were transfected with the following plasmid DNA and stained with polyspecific anti-BVDV calf antiserum DSV229/2 by the IPMA method. (1) pCMV- synE2.1; (2) pCMV_synE2.2.; (3) pCMVLP_synE2.1; (4) pCMV-LP_synE2.2.

motifs. The amplicon (AJ715399) was digested with HindIII and Sfil and subcloned inframe at the $5^{\prime}$ end of the optimised E2 sequences thus creating pLPsynE2.1 and pLP-synE2.2. The chimerical sequences were assigned accession numbers AJ715395 and AJ715396 respectively. The new constructs directed high production of the E2 protein as detected by bovine antisera on transfected COS cells (Fig. 1).

Trans-membrane anchor hydrophobic helices were detected with the TMHMM programme (not shown) and transfected COS cells were tested in conditions where membranes are preserved. Our recombinant E2 were thus considered membrane proteins.

Next, transfected COS cells were tested with a series of anti-BVDV calf antisera (Tab. III). Recognition paralleled the dis- tinction between BVDV-1 and BVDV2. Sera from calves infected with various BVDV-1 strains recognised COS cells transfected with pLP-synE2.1 but not COS cells transfected with pLP-synE2.2. Conversely anti-BVDV-2 sera recognised COS cells transfected with pLP-synE2.2 but not with pLP-synE2.1. A very strong crossrecognition was observed with antisera from calves that had been vaccinated with a BVDV-1 inactivated vaccine and challenged one month later with BVDV-2 thrombocytopenic strain 890 .

Finally, we investigated the cellular localisation of E2.1 and E2.2. Subconfluent COS7 cells were transfected in parallel with plasmid constructs expressing (i) glycoprotein $\mathrm{gD}$ from BoHV-1 (pCI$\mathrm{gD}$ ), (ii) protein $\mathrm{N}$ of BRSV (pNsyn), and (iii) E2 from BVDV-1 (pCMV-synE2.1) 
Table III. Specific recognition of BVDV-1 and BVDV-2 E2 by bovine antisera. Reactivity to type I and type E2 was detected on COS cells transfected with sequence-optimised constructs using antiBVDV antisera and anti-bovine secondary antibody conjugated to peroxydase. BVDV strains and bovine antisera indicated here have been characterised before [15, 24, 41]. Serum DSV 229/2 comes from an animal vaccinated with the Mucobovin ${ }^{\mathrm{TM}}$ vaccine then challenged with strain 890/256 [24]. Mouse monoclonal antibody 8H5-111 was developed against strain New-York-I.

\begin{tabular}{lccc}
\hline Antiserum & BVDV strain (genotype) & LP_E2.1 & LP_E2.2 \\
\hline CSV 229/1 & NADL (1a) & P. & N. \\
CSV 229/2 & BVR 1199 (1a) & P. & N. \\
2269 & Marloie (1b) & P. & N. \\
8H5-111 & New-York (1b) & P. & N. \\
ASV 545/6 & CULI 4 (1d) & P. & N. \\
ASV 684/1 & L256 (1f) & P. & N. \\
ASV 620 & L256 (1f) & PP. & p. \\
DSV 229/2 & Averonite (BDV), New-York (1b)+890 (2) & PP. & PP. \\
BSV 570 & 890 (2) & N. & P. \\
2270 & WVD829 (2) & N. & P. \\
\hline
\end{tabular}

N. Negative; p. weak positive; P. positive; PP. strong positive.

and BVDV-2 (pCMV-synE2.2). After $48 \mathrm{~h}$ incubation, cells in permeabilised and nonpermeabilised conditions were incubated with the respective specific antisera according to the IPMA method. The results are shown in Figure 2. As expected, COS cells transfected with plasmid pCI-gD appeared stained in non-permeabilised and permeabilised conditions whereas cells transfected with pCMV-N were found stained in the permeabilised condition only. A large number of cells transfected with plasmids expressing E2.1 and E2.2 were stained in the permeabilised condition but, surprisingly, a proportion of them were also positive in the nonpermeabilised condition. We concluded that E2 is directed to the cell surface in some of the transfected cells.

Constructs pLP-synE2.1 and pLPsynE2.2 were thus selected for DNA vaccination experiments in cattle.

\subsection{DNA vaccination elicited a cross- neutralising humoral response}

Pooled pLP-synE2.1 and pLP-synE2.2 plasmid DNA was given by the IM, IN and ID routes simultaneously. Neutralising antibodies were monitored on BVDV1a strain NADL. One animal showed a transient low-level response (titre $=2$ ) at week 2 after the second DNA inoculation. All three vaccinated animals seroconverted after the third injection, indicating that immunity towards heterologous strains (i.e. not the ones from which the E2 sequences had been cloned) had been obtained. The sera also neutralised Osloss (BVDV-1b) and 890 (BVDV-2) strains, indicating that the response concerned the wider range of BVDV. We noticed a lower neutralisation titre towards 890 and NADL as compared to Osloss. The one control animal remained seronegative throughout the experiment, thus excluding concomitant pestivirus contamination (Tab. IV).

\section{DISCUSSION}

We report DNA immunisation experiments in cattle using plasmid constructs that encode full-length versions of sequence-optimised glycoprotein E2 from BVDV-1 (E2.1) and BVDV-2 (E2.2). 

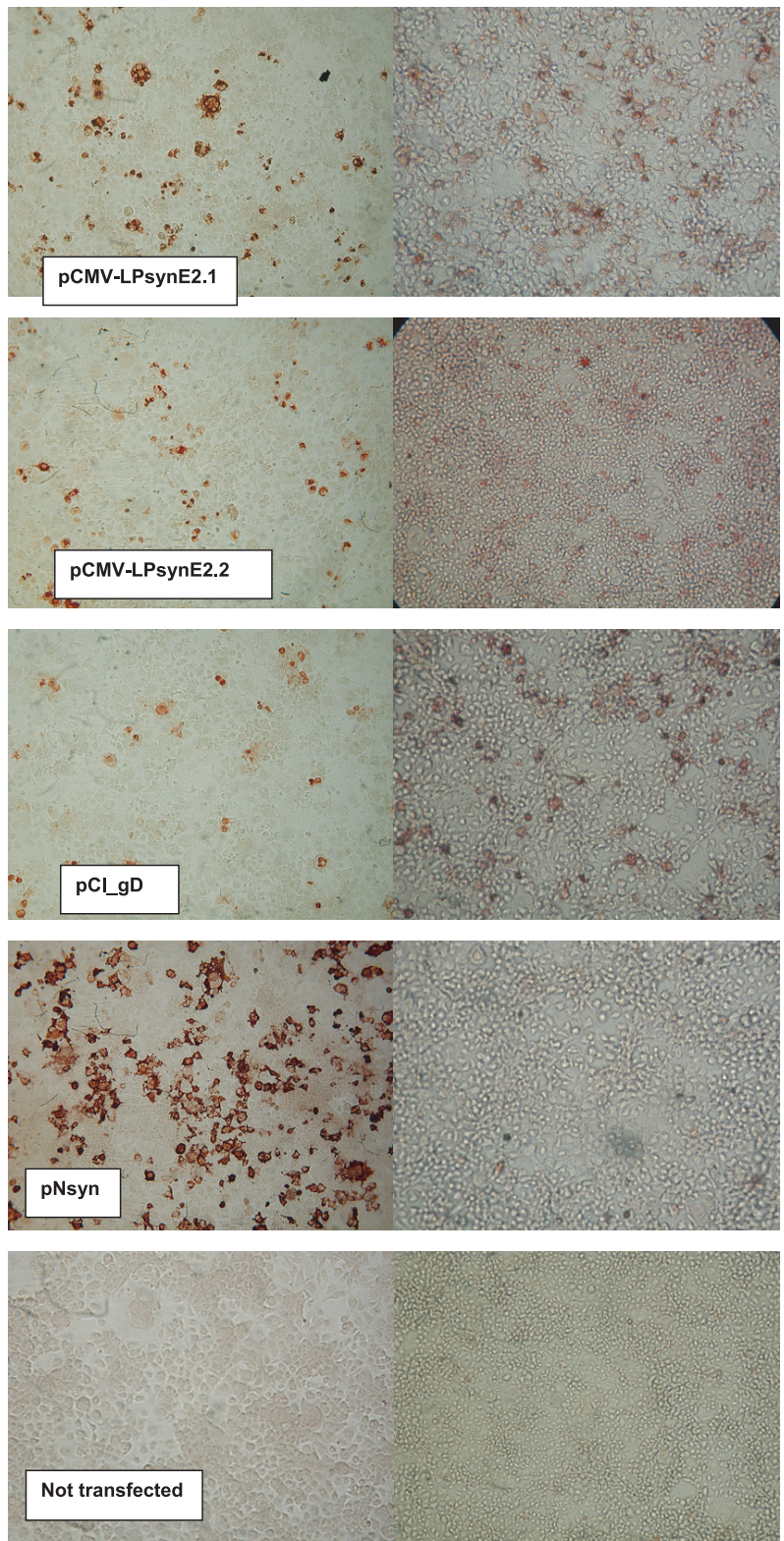

Figure 2. Localisation of recombinant proteins in transfected COS7 cells. COS cells in 24-well plates were transfected with plasmid constructs expressing E2 from BVDV-1b (pCMV-LPsynE2.1), E2 from BVDV-2 (pCMV-LPsynE2.2), surface protein gD from BoHV1 (pCI_gD) and the nucleocapsid N protein from BRSV (pNsyn). Non-transfected cells were included as controls. After $48 \mathrm{~h}$ at $37^{\circ} \mathrm{C}$, half the culture plates were incubated with the respective specific bovine antisera the permeabilised (left column) or non-permeabilised condition (right column) using the IPMA method. Non-transfected cells were incubated with the anti-E2 antiserum only. 
Table IV. Neutralising titres against representative BVDV strains after DNA immunisation. Three BVDV-negative crossbred bulls were inoculated with pooled pLP-synE2.1 and pLP-synE2.2 DNA by the combined IM, ID and IN routes and boosted twice by the combined IM and ID routes. One bull was kept non-vaccinated as an environmental control. Sera were analysed for neutralising antibodies two weeks after the second boost.

\begin{tabular}{lccccccc}
\hline Animal $N^{\circ}$ & $\begin{array}{c}\text { NADL } \\
(1 \mathrm{a})\end{array}$ & $\begin{array}{c}\text { Osloss } \\
(1 \mathrm{~b})\end{array}$ & $\begin{array}{c}\text { Culi4 } \\
(1 \mathrm{~d})\end{array}$ & $\begin{array}{c}\text { L256 } \\
(1 \mathrm{e})\end{array}$ & $\begin{array}{c}\text { WVD829) } \\
(2)\end{array}$ & $\begin{array}{c}\text { BSE921 } \\
(2)\end{array}$ & $\begin{array}{c}890 \\
(2)\end{array}$ \\
\hline 1 (vaccinated) & 16 & 256 & 64 & 128 & 32 & 16 & 64 \\
2 (vaccinated) & 16 & 512 & 128 & 64 & 8 & 8 & 16 \\
3 (vaccinated) & 32 & 512 & 128 & 64 & 16 & 16 & 32 \\
4 (unvaccinated) & $<2$ & $<2$ & $<2$ & $<2$ & $<2$ & $<2$ & $<2$ \\
\hline
\end{tabular}

At first, no protein expression was detected from plasmid constructs containing the native E2 sequences downstream of the strong CMV promoter in plasmid pCDNA3 (data not shown). The sequences were shown to contain internal splice sites, and a polyadenylation motif as well as an "instability" (ATTTA) motif (Tab. II). The codon usage was also found to be very divergent from the codon usage of mammalian cells. We then constructed synthetic E2 genes removing unwanted motifs and changing the codon usage after a table of highly expressed human genes. A low level of the E2 protein in COS cells was then detected by bovine antisera but only from the E2.2 construct. A modified version of the leader peptide from BoHV1 protein $\mathrm{gD}$ was then inserted inframe to the 5' end of both E2.1 and E2.2. The new constructs were strongly recognised by sera from calves that had recovered from BVDV infection.

High levels of expression were obtained with optimised E2 coding sequences downstream of a modified gD leader peptide. Removing internal splice sites as well as polyadenylation and $\mathrm{A}-\mathrm{T}$ rich stretches ("instability" motifs) probably resulted in an increased stability and thus increased steady-state amounts of the specific messengers. Changing codon frequencies after a table of highly expressed human genes probably increased the levels of transla- tion of individual mRNA molecules thanks to a better availability of the tRNA pool. Finally, efficient export to the membrane meant that relevant neutralising epitopes were (better) exposed. In the field of DNA vaccination, this approach is often used successfully for various types of antigens [2]. The various types of modifications applied here have recently been shown to act synergistically in improving the success of an HIV gp120 DNA vaccine [59].

Because E2 is initially part of the BVDV polyprotein, which is then processed into individual structural proteins during the BVDV infection cycle [56], it does not possess its own leader peptide, which therefore must be added experimentally. Other authors had previously used the signal peptide from a mouse IgG $\kappa$ light chain [45], from gD of BoHVI [3, 42], tPA [42] or from BVDV Erns [52]. In the early E2 construct of Harpin et al. [26], the 3' end of the E1 coding sequence was retained at the 5' end of the E2 coding sequence. In the expressed recombinant E2, the 16-residues C-terminal hydrophobic stretch of protein E1 probably acts as a signal peptide. We chose gD because sequence analysis as well as experimental data of our research team [55] indicated that it is highly expressed and efficiently directs membrane export of the protein. Its coding sequence was modified to permit 
straightforward cloning at the 5' end of E2 while keeping the native amino-terminal end of the E2 protein after cleavage.

The observation that only cells expressing E2 from constructs containing the leader peptide are strongly recognised by bovine antisera suggests that proper processing and/or membrane export is essential (Fig. 1). Differential recognition of E2.1 and E2.2 recombinant protein by antisera from calves infected with BVDV-1 or BVDV-2 strains (Tab. III) further indicated that native antigenic determinants were conserved.

Finally, the cellular localisation of E2 was investigated by IPMA on transfected $\mathrm{COS}$ cells in permeabilised and nonpermeabilised conditions (Fig. 2). We considered IPMA as a reliable approach because Toussaint et al. [55] in this research group, showed that their results are consistent with confocal microscopy. We thus compared the localisation of E2 with bona fide surface protein $\mathrm{gD}$ of $\mathrm{BoHV}-1$ and cytoplasmic protein $\mathrm{N}$ of BRSV. As expected $\mathrm{gD}$, but not $\mathrm{N}$, was observed at the cell surface. A proportion of COS cells transfected with pCMV-LPsynE2.1 and pCMVLPsynE2 expressed E2 at the cell surface. This was surprising because glycoprotein E2 is normally not transported to the cell surface but retained in the endoplasmic reticulum [34]. This is due to the presence of an arginine residue within the membrane anchor of E2 [34]. The arginine residue is also observed in the amino-acid sequence of membrane anchors of our recombinant E2. However, surface localisation of E2 has also been observed in cells infected with recombinant baculovirus [39] or recombinant VSV [21] that express E2. As suggested by Köhl et al. [34,35] it is conceivable that, in some transfected cells, the cell retention machinery becomes saturated by overexpressed E2 glycoproteins which are then transported to the cell surface.
The two E2 constructs (pCMVLPsynE2.1 and pCMV-LPsynE2.2) were injected into 3 bulls by the combined IM, ID and IN routes. All animals seroconverted after three injections of $1 \mathrm{mg}$ pooled DNA. Seroneutralisation experiments were performed on a total of 4 strains from various BVDV-1 subtypes and 3 BVDV-2 strains. They were laboratory strains (NADL, Osloss), recent field isolates from PI animals (Culi4, L256, WVD829, BSE921) and a virulent strain (890), a cytopathogenic strain (NADL) and several non-cytopathogenic ones. All were neutralised by sera from bulls after DNA immunisation (Tab. IV).

We concluded that our E2 constructs were immunogenic to the bovine species and elicited a cross-reactive humoral response to the wider range of BVDV strains. i.e. not only the ones from which the coding sequences had been cloned.

In recent years, several research teams have reported DNA immunisation to BVDV structural proteins in the mouse [26, 42, 45, 46] and cattle [27, 47, 42]. All experiments involved E2 constructs from a single BVDV-1a viral strain. In most reports, cross-neutralisation with strains from other genotypes was not tested. Harpin et al. [26, 27] showed that $3 / 4$ vaccinated mice cross-neutralised BVDV-1b strain New-York as well as BVDV-1a NADL but not BVDV-2 strain 125 , indicating that cross-neutralisation is restricted to the genotype from which the coding sequence had been cloned. Another report by Nobiron et al. [46] showed that the response to heterologous strain NADL was much lower in terms of titres and numbers of responding mice than to homologous strain Ky1203nc though both belong to the same genotype Ia. In cattle, the antibodies neutralised only homologous strain Ky1203nc but not NADL [47]. This difference could be due to differences in recombinant E2. Indeed, whereas Harpin's construct encodes a 
full-length E2 including its predicted native cleavage site and hence native N-terminus, Nobiron's is deleted at the C-terminus and uses an immunoglobulin signal peptide with its own cleavage site thus creating a truncated E2 protein with a chimeric N-terminus (the first 17 residues of the mature protein are from the multiple cloning site of vector pSecTag). Although titres were higher with the truncated E2 construct, cross-neutralisation of strains within genotype Ia was absent or poor even when genetic adjuvant IL-2 and GM-CSF were co-inoculated $[45,46]$. Accordingly, cross-neutralisation of BVDV strains from the same viral species depended on the integral E2 protein which should include its native $\mathrm{N}$-terminus. Besides, neutralisation of viral strains across the genotype divide is not observed when using E2 constructs from BVDV-1 solely.

When testing BVDV-1 strains, we observed that neutralising titres were lower towards NADL and higher to Osloss. They did not differ amongst BVDV-2 strains. The amino-acid sequences of E2 from NADL and Osloss are $71 \%$ and $84 \%$ identical, respectively, to injected E2.1. Besides, the E2 of BVDV-2 strain WVD829 is $100 \%$ identical to injected E2.2 whereas E2 from the other BVDV-2 strains tested are only $91 \%$ identical to injected E2.2. This suggested that genetic distance alone does not explain the observed differences in titres. The cytopathogenic (CP) or noncytopathogenic (NCP) biotype or other properties of testing strains might play a role. Finally, when comparing only NCP strains from the two species, titres were lower towards BVDV-2 strains than towards BVDV-1. This suggested that differences in immunogenicity or expression levels of the two E2 proteins might also play a role.

Recombination between the viral genome and cellular or viral RNA is known to occur in pestiviruses including BVDV. It may lead to the appearance of the cytopathogenic biotype in noncytopathogenic strains [38] and it is suspected to generate variability [31]. Therefore the risk of in vivo recombination between an injected DNA vaccine (plasmids) and a field BVDV isolate is worth considering. First, cell lines continuously expressing structural proteins including E2 cannot be superinfected with BVDV $[25,48]$ and E2 was recently shown to be responsible for blocking the entry of superinfecting BVDV [40]. Therefore bovine cells expressing E2 after DNA vaccination probably have a very low chance of being infected by the virus. Admittedly, DNA vaccination in PI calves might lead to viral genomic RNA and RNA from the injected plasmid being present in the same cells but, since PI calves are normally screened for and culled, this would be very infrequent. Secondly, in order to generate viable viruses, non-homologous recombination between messengers from the injected plasmid constructs and the viral genome will have to be inframe and not lead to disruption of the coding sequences for essential viral proteins. The changed $\mathrm{G}$ and $\mathrm{C}$ content of the $\mathrm{E} 2$ coding sequences in the injected constructs will greatly reduce the chances of homologous recombination. Homologous recombination is a minor mechanism for BVDV but it has been documented [19, 31]. Third, viable virus expressing E2 from the DNA vaccine will probably be neutralised by anti-E2 antibodies elicited by the DNA immunisation itself. Acquisition of a $\mathrm{CP}$ biotype due to insertion of E2 sequences has not been documented so far [38]. We conclude that the danger associated with the recombination between a DNA vaccine expressing E2 and a field BVDV isolate is probably very small.

In conclusion, we have shown here that sequence-optimised E2 glycoproteins were efficiently expressed in vitro and presented native antigenic determinants. 
Cross-neutralising antibodies were elicited in bulls when inoculated with pooled plasmid DNA. This is a promising first step to making a cross-protective DNA vaccine against BVDV. It remains to be seen whether our E2 can lead to effective protection against viral challenge. The most efficient inoculation route should be determined too. Because of demonstrated immunogenicity and high levels of expression they achieved, our constructs may also be useful to prepare recombinant proteins to be included in a subunit vaccine.

\section{ACKNOWLEDGEMENTS}

We thank Dr Bolin (NADC, Ames USA) for providing the BVDV 890/256 viral strain, Dr J.F. Toussaint for the gift of the pCI$\mathrm{gD}$ plasmid, Mr M. Boxus for the pNsyn plasmid. The expert technical assistance of M. Loncar, G. Vandendaele, D. Léglise, L. Rosaer, F. Stockmans and workers of the VAR isolation unit in Machelen is gratefully acknowledged. This research was supported by contract No. S-6041 from Federal Public Service Health, Food chain safety and Environment of the Belgian Federal Government.

\section{REFERENCES}

[1] Baker J.C., The clinical manifestations of bovine viral diarrhea infection, Vet. Clin. North Am. Food Anim. Pract. (1995) 11:425-445.

[2] Barouch D., Rational design of gene-based vaccines, J. Pathol. (2006) 208:283-289.

[3] Baxi M.K., Deregt D., Robertson J., Babiuk L.A., Schlapp T., Tikoo S.K., Recombinant bovine adenovirus type 3 expressing bovine viral diarrhea virus glycoprotein E2 induces an immune response in cotton rats, Virology (2000) 278:234-243.

[4] Becher P., Orlich M., Thiel H.J., Complete genomic sequence of border disease virus, a pestivirus from sheep, J. Virol. (1998) 72:5165-5173.

[5] Bevilacqua A., Ceriani M.C., Capaccioli S., Nicolin A., Post-transcriptional regulation of gene expression by degradation of messenger RNAs, J. Cell. Physiol. (2003) 195:356372 .
[6] Bolin S.R., Immunogens of bovine viral diarrhea virus, Vet. Microbiol. (1993) 37:263271.

[7] Bolin S.R., Ridpath J.F., Glycoprotein E2 of bovine viral diarrhea virus expressed in insect cells provides limited protection from systemic infection and disease, Arch. Virol. (1996) 141:1463-1477.

[8] Boxus M., Tignon M., Roels S., Toussaint J.F., Walravens K., Benoit M.A., Coppe P., Letesson J.J., Letellier C., Kerkhofs P., DNA immunization with plasmids encoding fusion and nucleocapsid proteins of BRSV induces a strong cell-mediated immunity and protects calves against challenge, J. Virol. (2007) 81:6879-6889.

[9] Boulanger D., Waxweiler S., Karelle L., Loncar M., Mignon B., Dubuisson J., Thiry E., Pastoret P.P., Characterization of monoclonal antibodies to bovine viral diarrhoea virus: evidence of a neutralizing activity against gp48 in the presence of goat antimouse immunoglobulin serum, J. Gen. Virol. (1991) 72:1195-1198.

[10] Brunak S., Engelbrecht J., Knudsen S., Prediction of human mRNA donor and acceptor sites from the DNA sequence, J. Mol. Biol. (1991) 220:49-65.

[11] Bruschke C.J., Moormann R.J., van Oirschot J.T., van Rijn P.A., A subunit vaccine based on glycoprotein E2 of bovine virus diarrhea virus induces fetal protection in sheep against homologous challenge, Vaccine (1997) 15:1940-1945.

[12] Bruschke C.J., van Oirschot J.T., van Rijn P.A., An experimental multivalent bovine virus diarrhea virus E2 subunit vaccine and two experimental conventionally inactivated vaccines induce partial fetal protection in sheep, Vaccine (1999) 17:1983-1991.

[13] Collen T., Morrison W.I., CD4(+) T-cell responses to bovine viral diarrhoea virus in cattle, Virus Res. (2000) 67:67-80.

[14] Collen T., Carr V., Parsons K., Charleston B., Morrison W.I., Analysis of the repertoire of cattle CD4(+) T cells reactive with bovine viral diarrhoea virus, Vet. Immunol. Immunopathol. (2002) 87:235-238.

[15] Couvreur B., Letellier C., Collard A., Quenon P., Dehan P., Hamers C., Pastoret P.P., Kerkhofs P., Genetic and antigenic variability in bovine viral diarrhea virus (BVDV) isolates from Belgium, Virus Res. (2002) 85:17-28.

[16] De Moerlooze L., Lecomte C., BrownShimmer S., Schmetz D., Guiot C., Vandenbergh D., Allaer D., Rossius M., Chappuis G., Dina D., et al., Nucleotide sequence of the bovine viral diarrhoea 
virus Osloss strain: comparison with related viruses and identification of specific DNA probes in the 5' untranslated region, J. Gen. Virol. (1993) 74:1433-1438.

[17] Elahi S.M., Shen S.H., Talbot B.G., Massie B., Harpin S., Elazhari Y., Recombinant adenoviruses expressing the E2 protein of bovine viral diarrhea virus induce humoral and cellular immune responses, FEMS Microbiol. Lett. (1999) 177:159-166.

[18] Elouahabi A., Thiry M., Pector V., Ruysschaert J.M., Vandenbranden M., Calorimetry of cationic liposome-DNA complex and intracellular visualization of the complexes, Methods Enzymol. (2003) 373:313-332.

[19] Gallei A., Orlich M., Thiel H.-J., Becher P., Noncytopathogenic pestivirus strains generated by nonhomologous recombination: alterations in the NS4A/NS4B coding region, J. Virol. (2005) 79:14261-14270.

[20] Gregory D., Tascon R.E., Lowrie D.B., Repeated use of Qiagen columns in largescale preparation of plasmid DNA, in: Lowrie D.B., Whalen R.G. (Eds.), Methods in molecular medicine, vol. 29, DNA vaccines: methods and protocols, Humana Press Inc., Totowa, NJ, 2000.

[21] Grigera P.R., Marzocca M.P., Capozzo A.V., Buonocore L., Donis R.O., Rose J.K., Presence of bovine viral diarrhea virus (BVDV) E2 glycoprotein in VSV recombinant particles and induction of neutralizing BVDV antibodies in mice, Virus Res. (2000) 69:3-15.

[22] Haas J., Park E.C., Seed B., Codon usage limitation in the expression of HIV-1 envelope glycoprotein, Curr. Biol. (1996) 6:315324.

[23] Hamers C., Couvreur B., Dehan P., Letellier C., Lewalle P., Pastoret P.-P., Kerkhofs P., Differences in experimental virulence of bovine viral diarrhoea viral strains isolated from haemorrhagic syndromes, Vet. J. (2000) 160:250-258.

[24] Hamers C., Couvreur B., Dehan P., Letellier C., Fischer L., Brun A.J., Lewalle P., Michaux C., Kerkhofs P., Pastoret P.-P., Assessment of clinical and virological protection afforded by an inactivated BVDV-1 commercial vaccine against a BVDV-2 challenge, Vet. Rec. (2003) 153:236-240.

[25] Harada T., Tautz N., Thiel H.J., E2-p7 region of the bovine viral diarrhea virus polyprotein: processing and functional studies, J. Virol. (2000) 74:9498-9506.

[26] Harpin S., Talbot B., Mbikay M., Elazhari Y., Immune response to vaccination DNA encoding the bovine viral diarrhoea virus major glycoprotein gp53 (E2), FEMS Microbiol. Lett. (1997) 146:229-234.

[27] Harpin S., Hurley D., Mbikay M., Talbot B., Elazhari Y., Vaccination of cattle with a DNA plasmid encoding the bovine viral diarrhoea virus major glycoprotein E2, J. Gen. Virol. (1999) 80:3137-3144.

[28] Houe H., Epidemiological features and economical importance of bovine viral diarrhoea virus (BVDV) infections, Vet. Microbiol. (1999) 64:89-107.

[29] Howard C.J., Clarke M.C., Sopp P., Brownlie J., Immunity to bovine virus diarrhoea virus in calves: the role of different T-cell subpopulations analysed by specific depletion in vivo with monoclonal antibodies, Vet. Immunol. Immunopathol. (1992) 32:303314.

[30] Howard C.J., Clarke M.C., Brownlie J., Protection against respiratory infection with bovine virus diarrhoea virus by passively acquired antibody, Vet. Microbiol. (1989) 19:195-203.

[31] Jones L.R., Weber L., Homologous recombination in bovine pestiviruses: Phylogenetic and statistic evidence, Infect. Genet. Evol. (2004) 4:335-343.

[32] Kelling C.L., Evolution of bovine viral diarrhea virus vaccines, Vet. Clin. North Am. Food Anim. Pract. (2004) 20:115-129.

[33] Kozak M., The scanning model for translation: an update, J. Cell Biol. (1989) 108:229_ 241.

[34] Köhl W., Zimmer G., Greiser-Wilke I., Haas L., Moennig V., Herrler G., The surface glycoprotein E2 of bovine viral diarrhoea virus contains an intracellular localization signal, J. Gen. Virol. (2004) 85:1101-1111.

[35] Köhl W., Grone A., Moennig V., Herrler G., Expression of the surface glycoprotein E2 of Bovine viral diarrhea virus by recombinant vesicular stomatitis virus, J. Gen. Virol. (2007) 88:157-165.

[36] Kramps J.A., Magdalena J., Quak J., Weerdmeester K., Kaashoek M.J., MarisVeldhuis M.A., Rijsewijk F.A., Keil G., van Oirschot J.T., A simple, specific, and highly sensitive blocking enzyme-linked immunosorbent assay for detection of antibodies to bovine herpesvirus 1, J. Clin. Microbiol. (1994) 32:2175-2181.

[37] Krogh A., Larsson B., von Heijne G., Sonnhammer E.L.L., Predicting transmembrane protein topology with a hidden Markov model: application to complete genomes, J. Mol. Biol. (2001) 305:567-580.

[38] Kummerer B.M., Tautz N., Becher P., Thiel H., Meyers G., The genetic basis for cytopathogenicity of pestiviruses, Vet. Microbiol. (2000) 77:117-128. 
[39] Kweon C.H., Yoon Y.D., An S.H., Lee Y.S., Expression of envelope protein (E2) of bovine viral diarrhea virus in insect cells, J. Vet. Med. Sci. (1997) 59:415-419.

[40] Lee Y.M., Tscherne D.M., Yun S.I., Frolov I., Rice C.M., Dual mechanisms of pestiviral superinfection exclusion at entry and RNA replication, J. Virol. (2005) 79:3231-3242.

[41] Letellier C., Kerkhofs P., Wellemans G., Vanopdenbosch E., Detection and genotyping of bovine diarrhea virus by reverse transcription-polymerase chain amplification of the 5' untranslated region, Vet. Microbiol. (1999) 64:155-167.

[42] Liang R., Van den Hurk J.V., Zheng C., Yu H., Pontarollo R.A., Babiuk L.A., Van den Hurk S., Immunization with plasmid DNA encoding a truncated, secreted form of the bovine viral diarrhea virus E2 protein elicits strong humoral and cellular immune response, Vaccine (2005) 23:5252-5262.

[43] Liu M.A., DNA vaccines: a review, J. Intern. Med. (2003) 253:402-410.

[44] Nielsen H., Engelbrecht J., Brunak S., von Heijne G., Identification of prokaryotic and eukaryotic signal peptides and prediction of their cleavage sites, Protein Eng. (1997) 10:1-6.

[45] Nobiron I., Thompson I., Browlie J., Collins M.E., Co-administration of Il-2 enhances antigen-specific immune response with DNA encoding the glycoprotein E2 of bovine diarrhoea virus, Vet. Microbiol. (2000) 76:129142.

[46] Nobiron I., Thompson I., Browlie J., Collins M.E., Cytokine adjuvancy of BVDV DNA vaccine enhances both humoral and cellular immune response in mice, Vaccine (2001) 19:4226-4235.

[47] Nobiron I., Thompson I., Browlie J., Collins M.E., DNA vaccination against bovine diarrhoea virus induces humoral and cellular responses in cattle with evidence for protection against viral challenge, Vaccine (2003) 21:2082-2092.

[48] Reinman I., Meyers G., Beer M., Transcomplementation of autonomously replicating Bovine viral diarrhea virus replicons with deletion in the E2 coding region, Virology (2003) 307:213-227.

[49] Ridpath J.F., Bolin S.R., The genomic sequence of a virulent bovine viral diarrhea virus (BVDV) from the type 2 genotype: detection of a large genomic insertion in a noncytopathic BVDV, Virology (1995) 212:39-46.
[50] Ridpath J.F., Neill J.D., Frey M., Landgraf J.G., Phylogenetic, antigenic and clinical characterization of type 2 BVDV from North America, Vet. Microbiol. (2000) 77:145155.

[51] Rümenapf T., Unger G., Strauss J.H., Thiel H.J., Processing of the envelope glycoproteins of pestiviruses, J. Virol. (1993) 67:3288-3294.

[52] Schmitt J., Becher P., Thiel H.J., Keil G.M., Expression of bovine viral diarrhoea virus glycoprotein E2 by bovine herpesvirus-1 from a synthetic ORF and incorporation of E2 into recombinant virions, J. Gen. Virol. (1999) 80:2839-2848.

[53] Tajima M., The prevalent genotypes of bovine viral diarrhea virus in Japan, Germany and the United States of America, Jpn J. Vet. Res. (2006) 54:129-134.

[54] Toussaint J.F., Coen L., Letellier C., Dispas M., Gillet L., Vanderplasschen A., Kerkhofs P., Genetic immunisation of cattle against bovine herpesvirus 1: glycoprotein $\mathrm{gD}$ confers higher protection than glycoprotein $\mathrm{gC}$ or tegument protein VP8, Vet. Res. (2005) 36:529-544.

[55] Toussaint J.F., Letellier C., Paquet D., Dispas M., Kerkhofs P., Prime-boost strategies combining DNA and inactivated vaccines confer high immunity and protection in cattle against bovine herpesvirus-1, Vaccine (2005) 23:5073-5081.

[56] Van Regenmortel M.H.V., Fauquet C.M., Bishop E.B., Carstens E.B., Estes M.K., Lemon S.M., Maniloff J., Mayo M.A., McGeoch D.J., Pringle C.R., Wickner R.B., Virus taxonomy: classification and nomenclature of viruses, Seventh report of the International Committee on taxonomy of viruses, Academic Press, San Diego, 2000.

[57] Vilcek S., Durkovic B., Kolesarova M., Paton D.J., Genetic diversity of BVDV: consequences for classification and molecular epidemiology, Prev. Vet. Med. (2005) 72:31-35.

[58] Wang L., Sunyer J.O., Bello L.J., Fusion to C3d enhances the immunogenicity of the E2 glycoprotein of type 2 bovine diarrhea virus, J. Virol. (2004) 78:1616-1622.

[59] Wang S., Farfan-Arribas D.J., Shen S., Chou T.H., Hirsch A., He F., Lu S., Relative contributions of codon usage, promoter efficiency and leader sequence to the antigen expression and immunogenicity of HIV-1 Env DNA vaccine, Vaccine (2006) 24:45314540 . 